\title{
Does treatment with olmesartan improve arterial stenoses due to fibromuscular dysplasia?
}

\author{
Hypertension Research (2009) 32, 927-929; doi:10.1038/hr.2009.126; published online 28 August 2009
}

Fibromuscular dysplasia (FMD) represents a group of non-atherosclerotic, non-inflammatory arterial diseases that most commonly involve renal and carotid arteries. Histological classification discriminates three main subtypes (intimal, medial and perimedial), which may be associated in the same patient. Although the etiology of FMD is not well understood, several mechanisms have been proposed, such as genetic predisposition, hormonal factors, paucity of vasa vasorum, repeated microtrauma, inadequate hormonal background, $\alpha$-antitrypsin deficiency and arterial wall ischemia. ${ }^{1}$ The molecular biology of FMD is unclear. Pathology seems to be characterized by smooth muscle hyperplasia, elastic fiber destruction, fibrous tissue proliferation and arterial wall disorganization. Angiographic classification includes a multifocal type (with multiple stenoses) and the string-of-beads pattern (related to medial FMD), and tubular and focal types (which are not clearly related to specific histological lesions).

No data are available in literature about the remission of arterial stenoses during treatment of FMD. As suggested by the Fibromuscular Dysplasia Society of America, there are no treatment protocols for FMD, and management depends on which arteries are affected and on symptoms. When FMD produces minimal stenotic lesions, it may be managed conservatively by antiplatelet or anticoagulant therapy as prophylaxis against complications.

As described in a recent paper by our group, ${ }^{2}$ a case of FMD diagnosed in a 52-year-old hypertensive woman involving internal carotid arteries, celiac tripod and the mesenteric superior artery was treated with acetylsalicylic acid (100 mg daily), and for arterial hypertension with olmesartan (20 mg daily) and hydrochlorothiazide $12.5 \mathrm{mg}$ daily. At 14-month follow-up, blood pressure was normalized $(124 / 78 \mathrm{~mm} \mathrm{Hg})$, heart rate decreased from 88 to 64 b.p.m. and the patient stopped smoking; no neurological symptoms or angina abdominis were reported. An ultrasonography of the right internal carotid artery performed before treatment with olmesartan shows alternating regions of lumen narrowing (stenoses) and vessel dilation several centimetres distal to the carotid bifurcation (Figure 1a).

The bilateral carotid bruits found at the initial physical examination disappeared at follow-up; this finding led to a repeat neck ultrasound of extracranial vessels and to subsequent angiographic imaging. The patient underwent carotid ultrasonography, showing that the stenoses observed 14 months later were no longer present (Figure 1b).

Computed angiotomography of the arteries involved by FMD also showed that the string-of-beads pattern, the severe stenosis of the celiac tripod and the moderate stenosis of the mesenteric superior artery previously observed were no longer evident,

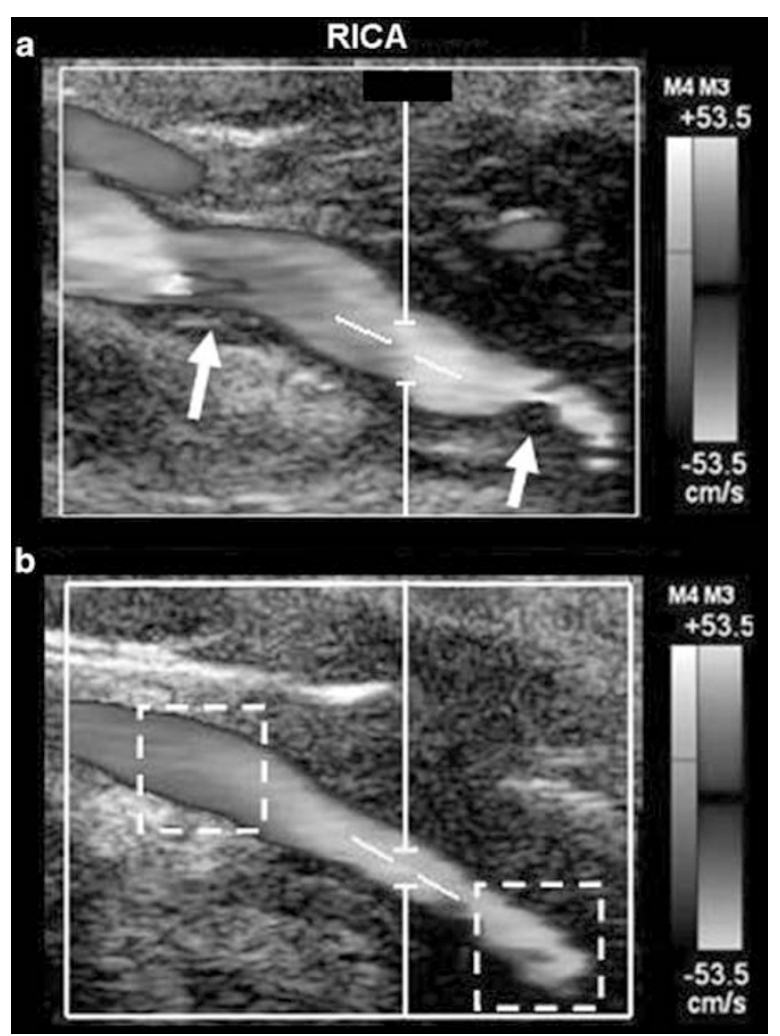

Figure 1 Ultrasonography of right internal carotid artery (RICA) before (a) and after (b) treatment with olmesartan. Stenoses of RICA (arrows) improved after treatment (dashed square). 
and the arterial wall became almost rectilinear, particularly in the right internal carotid artery (Figures 2 and 3). Laboratory tests confirmed slight normocitic anemia (hemoglobin $11.8 \mathrm{~g}$ per $100 \mathrm{ml}$ ) and hypercholesterolemia $(218 \mathrm{mg}$ per $100 \mathrm{ml})$, whereas the antinuclear antibodies title decreased (from $1: 140$ to $1: 40$ ) and extractable antinuclear antibodies, antineutrophil cytoplasmic antibodies, antiphospholipid antibodies, rheumatoid factor, C3 and C4 complement fraction and cryoglobulins remained normal. No change in coagulation status was observed before and after the beginning of medication.

C-reactive protein was in the normal range both before $(0.15 \mathrm{mg}$ per $100 \mathrm{ml})$ and after treatment $(0.11 \mathrm{mg}$ per $100 \mathrm{ml}$, n.v. $<0.50)$, supporting the opinion that FMD is a noninflammatory disease. After treatment with olmesartan, a slight increase in plasma renin activity (2.9 vs. $2.1 \mathrm{ng} \mathrm{ml}^{-1} \mathrm{~h}^{-1}$, n.v. $0.3-1.9$ ) and a decrease in plasma aldosterone (212 vs. $124 \mathrm{pg} \mathrm{ml}^{-1}$ ) were found, secondary to the physiological effect of angiotensin-II type I receptor $\left(\mathrm{AT}_{1}\right)$ blockers on the renin-angiotensin-aldosterone system (RAS).

These results raise important questions.

Did our patient really have FMD? The female gender and the young age of the patient, together with the radiological pattern of lesions and their presence in other vascular beds, hardly deny FMD. Furthermore, biochemical examinations excluded other illnesses producing non-atherosclerotic FMDlike stenoses, such as Ehlers-Danlos and Williams syndromes, or type 1 neurofibromatosis. ${ }^{1}$ Dyslipidemia and smoking are two strong risk factors for atherosclerosis. Our patient was a moderate smoker (5-7 cigarettes daily) and had mild hypercholesterolemia $(245 \mathrm{mg}$ per $100 \mathrm{ml}$ ) with high highdensity lipoprotein-cholesterol values $(69 \mathrm{mg}$ per $100 \mathrm{ml}$ ), this latter a well-established preventive factor for atherosclerosis. The diagnostic process differentiating FMD from atherosclerosis comes not from risk factors but from imaging techniques. It is usually not too difficult to differentiate between atherosclerosis and FMD, as the former generally occurs at the origin or proximal portion of the carotid artery in older subjects with classic cardiovascular risk factors, the latter in the middle or distal carotid artery in young subjects with moderate cardiovascular risk factors, such as in our patient. The hypertension observed in our patient when FMD was diagnosed could be related to the high doses of nimesulide taken for headache, and to the sudden cessation of smoking 4 days before observation. Essential hypertension is a multifactorial disease, the pathogenesis of which is often difficult to explain. Our patient probably had a common case of essential hypertension that arise in a young subject with a family history and sustained-tomoderate sympathetic overactivity due in part to a smoking habit. In agreement with this hypothesis, a slight increase in the plasma renin activity showing sympathetic overactivity was found at baseline $\left(2.1 \mathrm{ng} \mathrm{ml}^{-1} \mathrm{~h}^{-1}\right)$. Moreover, the $27 \%$ decrease in heart rate observed after treatment with olmesartan indirectly confirms the modulation of the sympathetic activity through $\mathrm{AT}_{1}$-blockade.

Another question is that if FMD was present at the first check, why did arterial stenoses improve? The pathophysiology and molecular biology of FMD are unknown, and this disease was listed by the National Organization of Rare Diseases only last year. If FMD causes abnormal cell development in the arterial wall, it is only natural to assume that endothelium has a role in its genesis. It is well established that endothelium contributes to maintain cardiovascular homeostasis, mainly through the activity of endotheliumderived nitric oxide (NO). However, in the presence of proatherogenic risk factors, including hypertension, diabetes mellitus, hypercholesterolemia and tobacco smoking, the bioavailability of NO is reduced. This condition, labeled as endothelial dysfunction, is characterized by vasoconstriction, platelet aggregation, leukocyte adhesion and smooth muscle cell proliferation.

Reduced availability of $\mathrm{NO}$ is mainly due to an increase in reactive oxygen species production, which in turn is responsible for NO breakdown. A large body of evidence indicates that, especially under pathological conditions, the activity of RAS is associated with angiotensin II-mediated reactive oxygen species production, thus unbalancing endothelial function and leading to progressive vascular disease. ${ }^{3}$ The action of the renin-angiotensin system is mostly linked to the downstream effects of binding with $\mathrm{AT}_{1}$-receptors, and its selective blockade restores endothelial function in patients with cardiovascular risk factors. Olmesartan, beyond its blood pressure-lowering effects, has been reported to affect the redox state of the vessel wall by restoring NO availability under different pathological conditions to exert anti-inflammatory effects and to increase the production of endothelial progenitor cells in diabetic subjects, ${ }^{4}$ in whom endothelial function is more injured than in hypertensives.

Although it is arduous to accept that $\mathrm{AT}_{1}$-blockers may have a pleyotropic effect

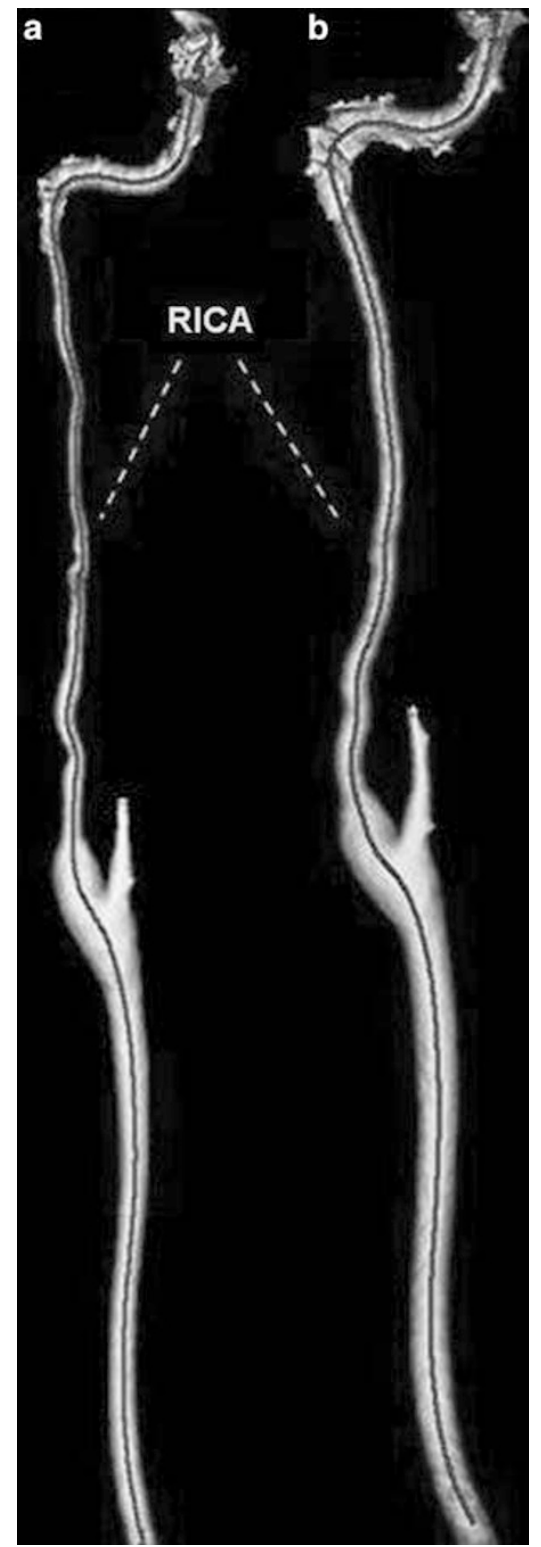

Figure 2 Three-dimensional cerebral computed angiotomography of right internal carotid artery (RICA) before (a) and after (b) treatment with olmesartan. Dashed lines show the string-ofbeads pattern that is typical of fibromuscular dysplasia.

that modifies the natural history of FMD, it is difficult as well to not attribute the modifications of arteries observed on imaging to medical treatment. Our results are not a completely new notation, as it has recently been shown that $\mathrm{AT}_{1}$-blockers are effective both in remodeling and in delaying the re-stenosis of renal arteries after angioplasty in subjects with FMD. ${ }^{5}$

The Fibromuscular Dysplasia Society of America is working together with the University of Michigan on an International Patient 


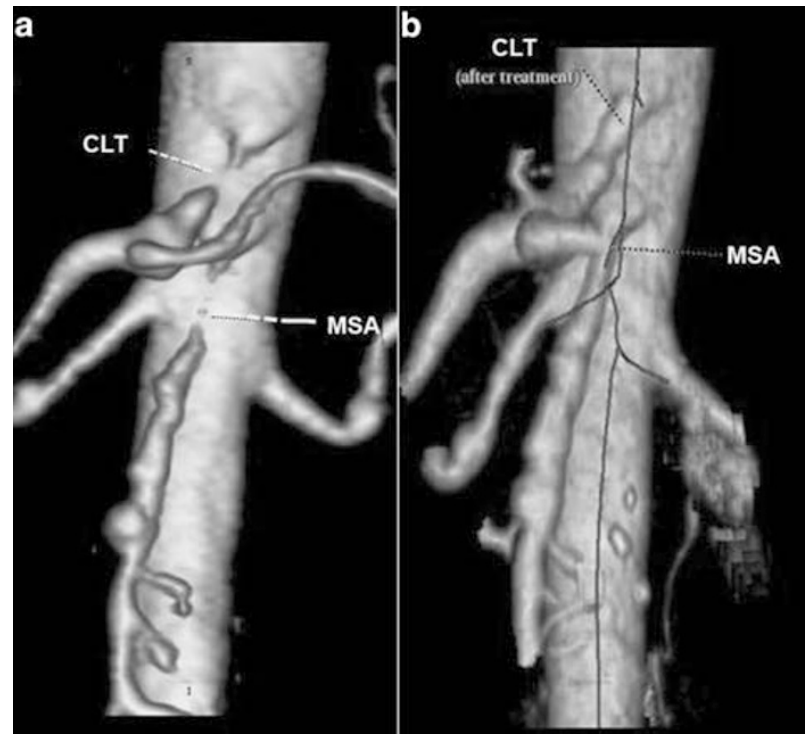

Figure 3 Three-dimensional computed angiotomography of abdominal vessels before (a) and after (b) treatment with olmsertan. Dashed lines show severe stenosis of the celiac tripod (CLT) and moderate stenosis of the mesenteric superior artery (MSA).

Registry, and the National Institute of Health is conducting a research on FMD to individualize its biological and molecular mechanisms. Some researchers of this society (http://www.fmdsa.org) suggest that FMD is probably due to an overactivity of the transforming growth factor- $\beta$ pathway, as shown in other non-atherosclerotic and non-inflammatory arterial diseases, for example, the Marfan's syndrome, a disorder caused by mutations in the gene that encodes fibrillin-1, determining aortic aneurysm and dissection. Habashi et $a .^{6}$ report that in a mouse model of Marfan's syndrome, aortic aneurysm can be prevented by transforming growth factor- $\beta$ antagonists such as transforming growth factor- $\beta$-neutralizing antibody or $\mathrm{AT}_{1}$-blockers administration. Particularly, losartan-based treatment prevented aortic aneurysms and marfanoid lesions, suggesting that in these two conditions, $\mathrm{AT}_{1}$-blocker effect is not only exerted on $\mathrm{AT}_{1}$ receptors but also on the transforming growth factor- $\beta$ pathway. This finding, first observed in an animal model, was recently confirmed in humans. In this respect, Brooke et al. ${ }^{7}$ reported that $\mathrm{AT}_{1}$-blockers significantly slowed the rate of progressive aorticroot dilation in a small cohort of pediatric patients with Marfan's syndrome. This effect is independent of blood pressure lowering, as no difference in blood pressure values was found after treatment. This suggests a potential efficacy of $\mathrm{AT}_{1}$-blockers in modifying the natural history of non-atherosclerotic arterial diseases such as FMD.

We hope that this case can help to stimulate further investigations with regard to FMD, a disease that has been reported to be the cause of sudden death in young people. $^{8}$
Alberto Mazza ${ }^{1}$, Stefano Cuppini ${ }^{1}$, Sergio Zamboni ${ }^{1}$, Laura Schiavon ${ }^{1}$, Luca Zattoni ${ }^{2}$, Andrea Viale ${ }^{2}$, Francesco Corbetti ${ }^{3}$, Roberta Ravenni ${ }^{4}$, Alberto Sacco $^{5}$ and Edoardo Casiglia ${ }^{6}$

${ }^{1}$ Department of Internal Medicine, General Hospital of Rovigo, Rovigo, Italy; ${ }^{2}$ Department of Imaging, General Hospital of Rovigo, Rovigo, Italy; ${ }^{3}$ Division of Radiology, Padova, Italy; ${ }^{4}$ Department of Neuroscience, General Hospital of Rovigo, Rovigo, Italy; 5 Department of Vascular Surgery, General Hospital of Rovigo, Rovigo, Italy and ${ }^{6}$ Department of Clinical and Experimental Medicine, University of

Padova, Padova, Italy

E-mail: mazza.alberto@azisanrovigo.it

1 Slovut DP, Olin JW. Fibromuscular dysplasia. N Engl J Med 2004; 350: 1862-1871.

2 Mazza A, Zamboni S, Cuppini S, Zattoni L, Ravenni R, Sacco A, Casiglia E. Internal carotid artery fibromuscular dysplasia in arterial hypertension: management in clinical practice. Blood Press 2008; 17: 274-277.

3 Ohtani K, Egashira K, Ihara Y, Nakano K, Funakoshi K, Zhao G, Sata M, Sunagawa K. Angiotensin II type 1 receptor blockade attenuates in-stent restenosis by inhibiting inflammation and progenitor cells. Hypertension 2006; 48: 664-670

4 Bahlmann FH, de Groot K, Mueller O, Hertel B, Haller H, Fliser D. Stimulation of endothelial progenitor cells: a new putative therapeutic effect of angiotensin II receptor antagonists. Hypertension 2005; 45: 526-529.

5 Tanemoto M, Takase K, Yamada T, Satoh A, Abe T, Ito S. Dilation of renal artery stenosis after administration of losartan. Hypertens Res 2007; 30: 999-1002.

6 Habashi JP, Judge DP, Holm TM, Cohn RD, Loeys BL, Cooper TK, Myers L, Klein EC, Liu G, Calvi C, Podowski M, Neptune ER, Halushka MK, Bedja D, Gabrielson K, Rifkin DB, Carta L, Ramirez F, Huso DL, Dietz HC. Losartan, an AT1 antagonist, prevents aortic aneurysm in a mouse model of Marfan sindrome. Science 2006; 312: 117-121.

7 Brooke BS, Habashi JP, Judge DP, Patel N, Loeys B, Dietz III HC. Angiotensin II blockade and aortic-root dilation in Marfan's syndrome. N Engl J Med 2008; 358: 2787-2795.

8 Brodsky SV, Ramaswamy G, Chander P, Braun A. Ruptured cerebral aneurysm and acute coronary artery dissection in the setting of multivascular fibromuscular dysplasia: a case report. Angiology 2007; 58: 764-767. 